\title{
Cultured Keratinocytes as Biological Wound Dressings
}

\author{
RICHARD J PYE \\ Cambridge
}

\begin{abstract}
Summary
Human epidermal keratinocytes now can be grown reliably and reproducibly in vitro to form multilayered epithelium. These sheets of cultured keratinocytes have been used successfully to autograft patients with severe burns, leg ulcers and following excision of extensive congenital naevi. Whilst the technique carries the obvious advantage of huge expansion of the initial skin biopsy, thus removing the need for painful and slow healing donor sites, problems have been encountered. The take rate has been lower than with conventional split skin grafts. The take rate can be increased by the provision of a dermis. This may be achieved by providing an allodermis or by the use of a highly meshed autologous split skin graft. The wound is then covered with autologous cultured keratinocyte grafts. Manufactured dermis has been under investigation for some years and animal work suggests this may be an alternative approach.

There is a delay of 2 to 3 weeks for culture of the autologous sheets of keratinocytes. This has led to the use of allogeneic grafts in a number of patients. The long term survival of these grafts has been attributed to the loss of antigen presenting cells during tissue culture. However some grafts have been rejected. Studies currently in progress may help resolve these anomalies.

Whilst a number of problems remain to be solved the technique of cultured keratinocyte grafting takes wound care into an exciting new era. Skin banks may now become more than a surgeon's dream.
\end{abstract}

Patients with full thickness burns require skin grafts in the early stages of treatment to reduce sepis, fluid loss and pain and to promote healing. The major problem in the treatment of extensively burned patients is the lack of suitable sites from which to take autologous skin grafts. Meshing of the grafts and repeated harvesting of the donor sites will increase the coverage but may be insufficient, and in the past this has been made good with pig skin, cadaveric allografts and the development of synthetic skin. However, all of these methods have considerable drawbacks.
An alternative treatment would be to increase the tissue available for grafting by growing the patient's own skin in vitro. The first attempts to culture skin were made in 1898 by Ljunggren and although subsequent workers improved the culture system, ${ }^{1}$ the technical problems remained formidable. A major advance occurred in 1975 when Rheinwald and Green succeeded in culturing keratinocytes from single-cell suspensions of human epidermal cells. ${ }^{2}$ The major advantage of this system is the amount of epithelium which can be grown in vitro. Starting from a 
small biopsy, $1 \mathrm{~cm}^{2}$ initially, a 100 fold amplification can be achieved in 2 weeks and up to a further 10 fold increase after subcultivation. The culture techniques, clinical applications and the development of these techniques are discussed.

\section{Culture techniques}

The Rheinwald and Green technique relies upon the provision of connective tissue products by a feeder cell layer of fibroblasts, which not only support keratinocyte culture but also inhibit overgrowth by the patients' fibroblasts. Keratinocytes are seeded at clonal density onto semiconfluent lethally irradiated $3 \mathrm{~T} 3$ transformed mouse embryo fibroblasts (Figs. 1 and 2). The culture medium contains a number of mitogens, fetal calf serum and epidermal growth factor. Each colony is initiated from a single 'basal epidermal cell'. The colonies are multilayered and consist of proliferating basal cells attached to the flask surface and differentiating cells which are more superficially placed (Fig. 3). Confluent mature cultures consist of an epithelial pellicle which can be enzymatically detached from the culture flask as an intact sheet and used for grafting 3 (Fig. 4).

Subsequently it was shown that by increasing the seeding density, altering the $\mathrm{pH}$ and incubating conditions, keratinocytes could be grown in the absence of a feeder layer and medium supplements. ${ }^{4}$ The technique has the advantage that feeder cells do not have to be cultured but the risk of fibroblast overgrowth is greater and amplification is considerably reduced.

More recently the development of the defined MCDB series of media has permitted serum free, clonal growth of keratinocytes with as few as 400 cells $/ \mathrm{cm}^{2}$. Optimisation of nutrients especially the reduction of calcium levels and the omission of fetal calf serum, favours proliferation and extends the life of the cultures. ${ }^{5}$ However it is a difficult and extremely time consuming medium to prepare.

Keratinocytes have been cultured on a number of substrates. However thick purified type I bovine collagen gel seeded with human fibroblasts produces a suitable lattice to act as a 'dermal equivalent'. ${ }^{6}$ Keratinocytes may be

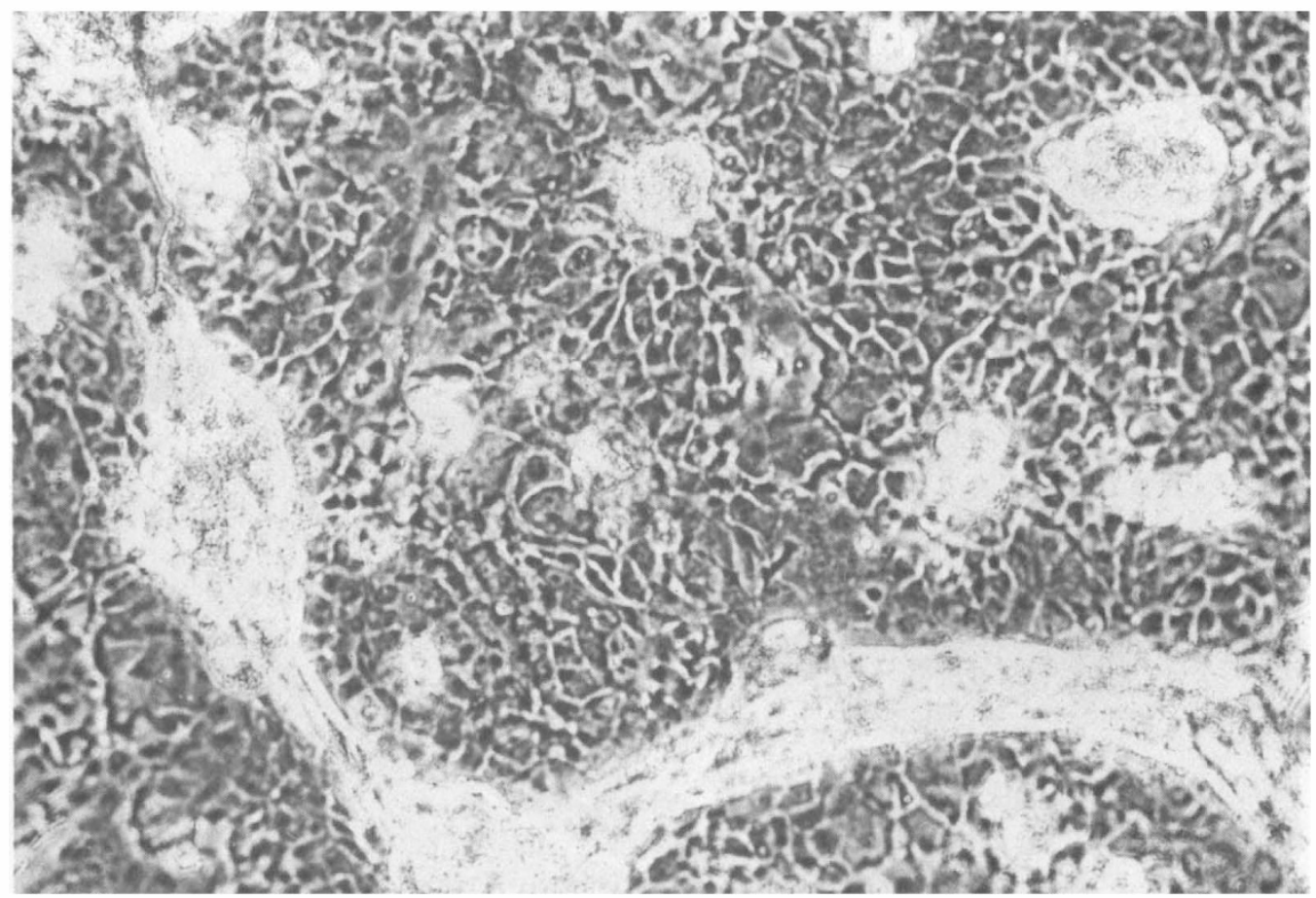

Fig. 1. Keratinocyte colonies displacing the lethally irradiated $3 T 3$ fibroblasts. 


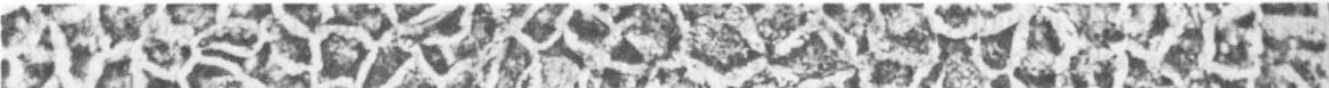

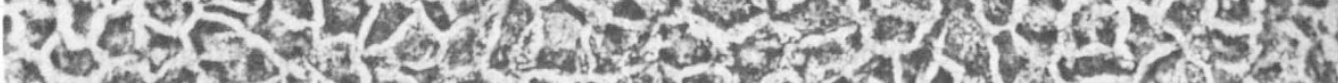

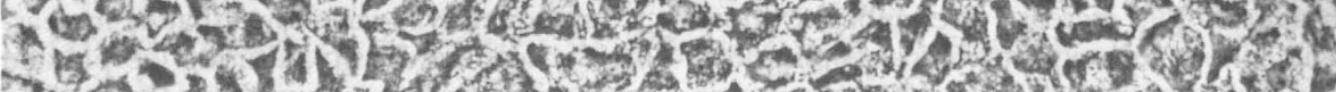

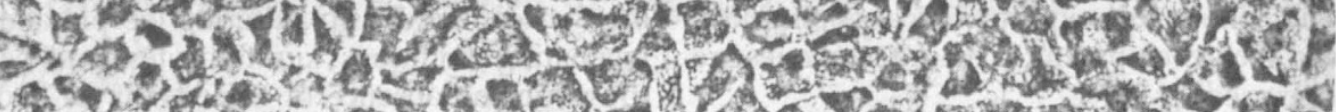

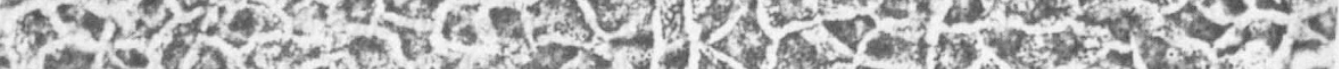

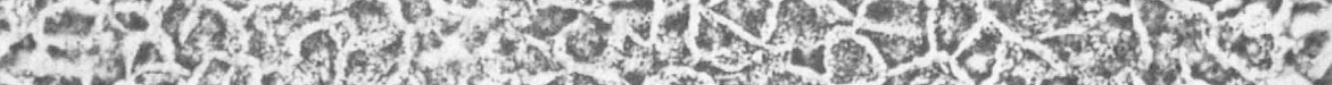

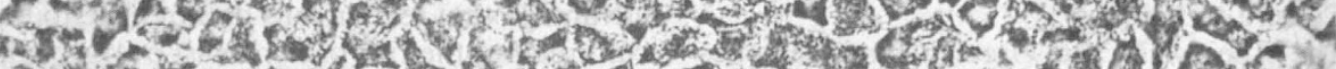

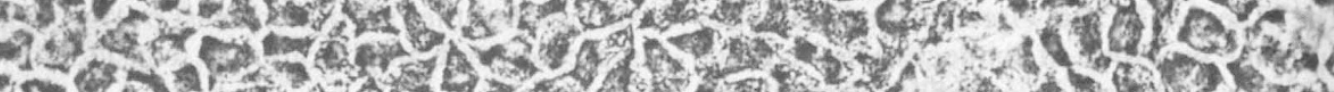
H.

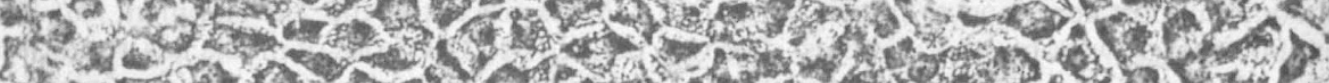

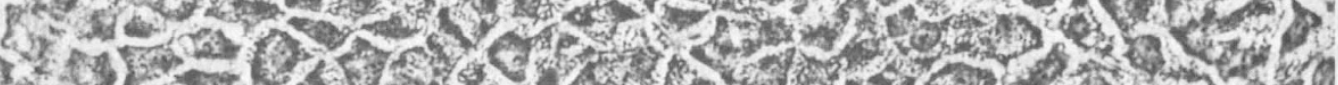

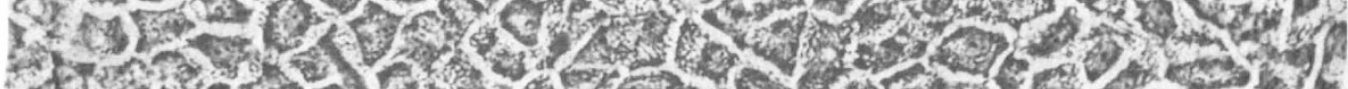

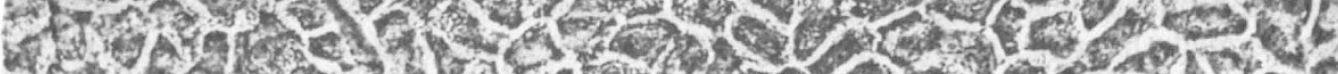

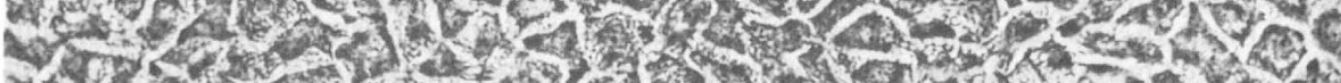

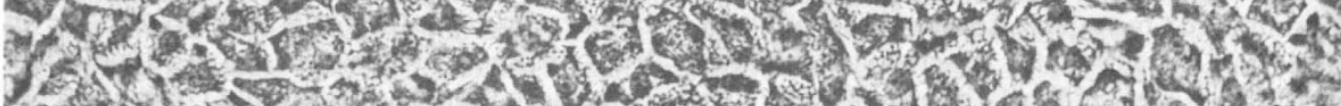

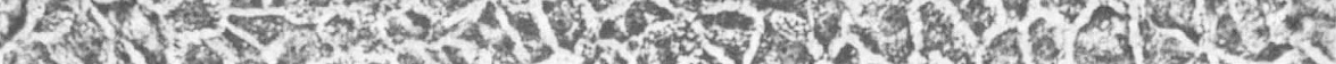

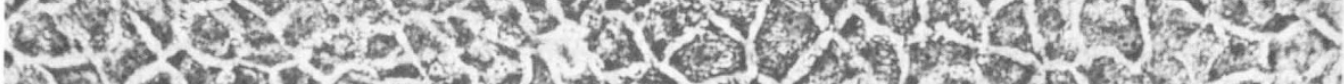

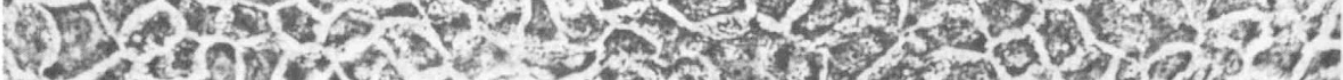

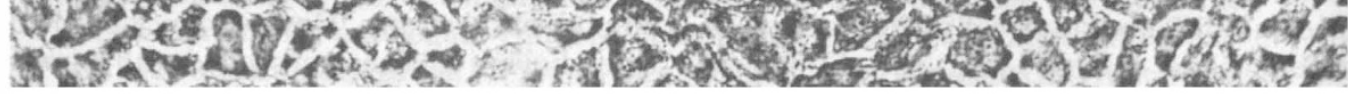

Fig. 2. A confluent sheet of keratinocytes suitable for grafting.

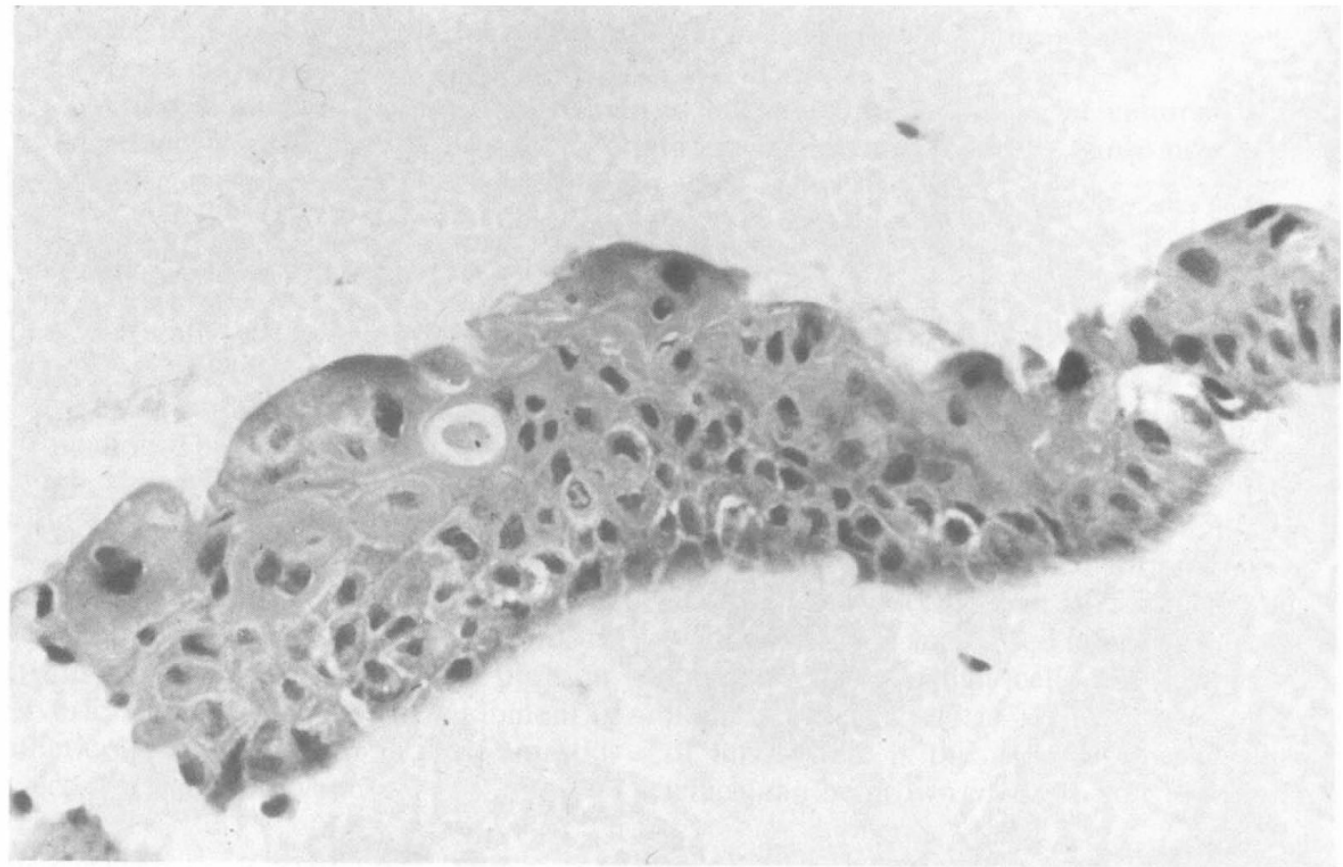

Fig. 3. A section through a cultured keratinocyte sheet. 


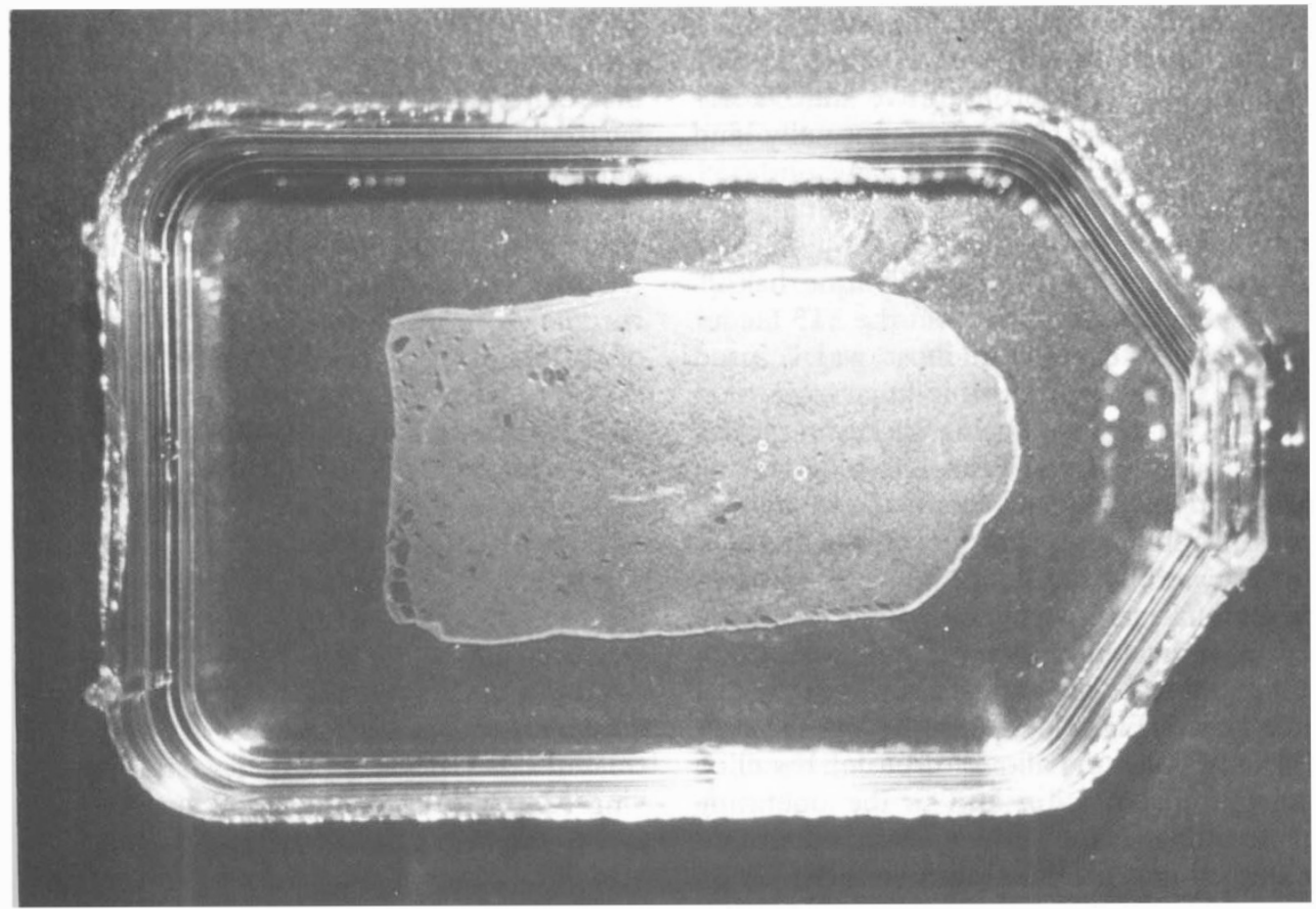

Fig. 4. A cultured keratinocyte sheet which has been enzyme digested off the plastic culture flask.

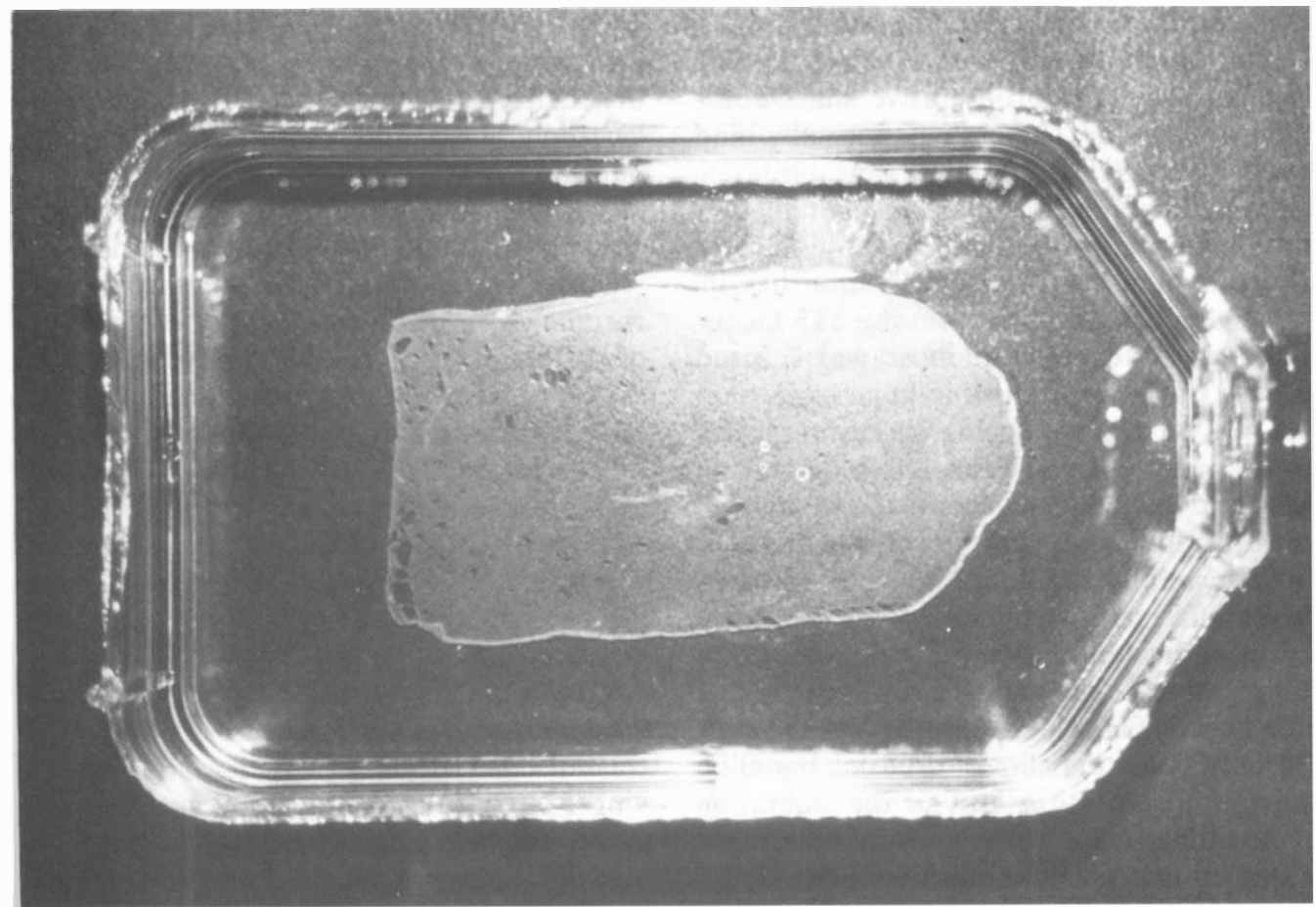

Fig. 5. A cultured keratinocyte sheet liga clipped to Vaseline gauze and ready for grafting. 
cultured either submerged in medium or at an air-interface. Under these latter conditions, although in a hyperproliferative state, keratinocytes appear to mature normally and closely resemble normal skin. ${ }^{7}$

Intact sheets of keratinocytes suitable for grafting can be produced using any of these culture systems and each technique has its ardent supporters. At present the 3T3 feeder cell layer system is the most widely used because of the reproducible huge expansion that can be achieved. Not all keratinocytes grow equally well in culture. Skin from ears, hands and feet is less successful than skin from breast, abdomen and prepuce. Growth rate is also affected by the donor's health and age. Sheets of keratinocytes even if grown on a dermal equivalent are very fragile and need to be clipped to a backing material such as Vaseline gauze (Fig. 5), N-terface or bridal veil. Not only does this allow for easier handling during transportation and in the operating theatre but also allows the grafts to be sutured or stapled into position on the patient.

\section{Applications}

Cultured keratinocyte grafting represents a major innovation in the management of wounds and has been used to treat burned patients, excised tattoos and elderly patients with leg ulcers or donor sites that are slow to heal. However all groups using cultured keratinocyte grafts have experienced a number of similar problems;

(i) an unexpectedly high number of grafts fail to take in spite of adequate preparation of the graft bed

(ii) an interval of at least 2 to 3 weeks is required from the time of the original biopsy before confluent sheets can be obtained in culture.

The low take rate is surprising since cultured keratinocyte sheets have an actively dividing layer of basal cells and if placed on a vascular bed should re-epithelialise the wound rapidly. However there are important differences between conventional split skin grafts and cultured keratinocyte grafts.

A conventional split skin graft contains blood vessels within the dermal elements and is fully keratinised. The capillaries 'link up' with vessels in the graft bed within a matter of 24 to 48 hours, ${ }^{8}$ serving both to secure the graft and to supply nutrients. The keratin layer protects the graft from drying. Although the cultured keratinocyte sheet is multilayered and terminally differentiated, it contains none of these elements and is therefore very vulnerable in the first 24 hours following grafting.

Low grade bacterial infection is common in chronic granulation tissue and therefore lysis of cultured keratinocyte grafts might be expected. However, the take rate is reduced even when this is excised and the graft placed on deep dermis, fat or deep fascia. The superficial dermis is known to play an important role in the control of keratinisation and may play a role in the protection of split skin grafts.

\section{Developments}

Cryopreserved split skin allografts have been used as a source of dermis. Cuono et al treated a patient who had thermal burns involving 55 per cent of the body surface, with cryopreserved cadaveric allografts from four separate donors. Subsequently the epidermis was stripped from the allograft using dermabrasion and the exposed allografted dermis was grafted with cultured autologous keratinocytes. The take rate was higher than would be anticipated without the allodermis'. Highly meshed split skin autografts (1-6) have been used in a similar way, but of course dermabrasion is unnecessary. The take rate appears to be greater and the final cosmetic appearance is superior to highly meshed split skin alone.

An alternative is to create an artificial dermis. A variety of substrates have been used. The collagen/fibroblast lattice has already been discussed. Although an interesting experimental model, it does not confer any real advantages when grafted onto patients and many grafts still fail to take. Perhaps the best known artificial skin is that devised by Yannas et al. ${ }^{10}$ The top layer is a conventional silicone elastomer, whilst the bottom layer is made up of highly porous covalently crosslinked purified bovine collagen and glycosaminoglycan (GAG). This bottom layer is degradable and acts as a tissue support which enhances the formation of a new dermis. The upper layer controls moisture loss, and although it is eventually ejected by the 
new dermis it can be removed at any stage. Keratinocytes either cultured or uncultured can be seeded on to the collagen-GAG layer of the membrane. To date these studies have only been undertaken in animals. It seems highly likely that this last approach will prove to be the most successful although the design of the composite membranes will almost certainly have to be very sophisticated.

The problem of the delay of supply of autologous cultured keratinocyte graft is due to the time required for culture, and has resulted in the examination of the use of allogeneic keratinocytes grown in vitro as grafts. Cultured foreign keratinocytes have been used successfully to allograft small areas in three patients with burns. There was no rejection and the grafts functioned well over a 9 month period. ${ }^{11}$ Twenty patients have now been treated without any apparent rejection of the grafts and other studies have confirmed these results. ${ }^{12}$

The survival of grafts of other organs such as thyroid, which have been cultured for a period in vitro is usually dramatically enhanced. This enhanced survival, which is sometimes permanent, is thought to be due to selective deaths of certain passenger cells in the graft which are essential for induction of the rejection response. ${ }^{13}$ These antigen presenting cells are also present in the skin where they are known as Langerhan's cells. These cells do not survive in these cultures. This interpretation is supported by the recent report of the long term survival of skin allografts in a massively burned child. Unrelated frozen cadaveric skin was used as allografts and the patient treated with Cyclosporin-A to stop early rejection of the grafts. The Cyclosporin-A was stopped at 120 days but the grafts remain stable without signs of rejection for a further 2 years. It is suggested that the freeze-thawing of the banked allografts may have eliminated the passenger antigen presenting cells. ${ }^{14}$

Keratinocytes grown in vitro using the techniques previously described are derived from single basal epidermal cells. There is no morphological evidence that Langerhans cells or lymphoid cells are present in these cultures. A graft of cultured keratinocytes, therefore, consists only of keratinocytes. There is no vascularisation within the graft and the basement membrane is formed by the keratinocytes in vivo.

However not all studies involving cultured keratinocyte allografts have shown long term survival of the grafts. Studies in pigs have shown that whilst there was a 7 day prolongation of cultured keratinocyte allograft survival, the grafts melted at 15 days post transplantation..$^{15}$ Recent work in man has also shown similar active rejection of cultured keratinocyte allografts. ${ }^{16}$

Further work is needed to explain the discrepancies between these studies. Do these allografts survive indefinitely or do they survive just long enough to allow the wounds to heal from the margins with the graft gradually being displaced?

Cultured keratinocytes can be stored long term in liquid nitrogen and resuscitated with a high plating efficiency. It is therefore quite feasible to establish a bank of autologous keratinocytes for an individual patient. There remain a number of difficulties to overcome but the technique of cultured keratinocyte grafts brings the establishment of universal skin banks a step nearer.

\section{References}

${ }_{1}^{1}$ Matoltsy AG: Epidermal cells in culture. Int Rev Cytol 1960, 10: 315-51.

${ }^{2}$ Rheinwald $\mathrm{J}$ and Green $\mathrm{H}$ : Serial cultivation of strains of human epidermal keratinocytes. Cell 1975: 6: 331-44.

${ }^{3}$ Gallico GC et al.: Permanent coverage of large burns wounds with autologous cultured epithelium. $N$ Eng J Med 1984, 311: 448-51.

${ }^{4}$ Eisinger $\mathrm{M}$ et al.: Human epidermal cell cultures: Growth and differentiation in the absence of dermal components or medium supplements. Proc Natl Acad Sci 1979, 76: 5340-4.

${ }^{5}$ Boyce S and Ham RG: Calcium-regulated differentiation of normal human epidermal keratinocytes in chemically defined clonal culture and serum free serial culture. J Invest Dermatol 1983, 81: $33 \mathrm{~s}-40 \mathrm{~s}$.

${ }^{6}$ Bell E et al.: The reconstitution of living skin. $J$ Invest Dermatol 1983, 81: 2s-10s.

${ }^{7}$ Asselineau D et al.: Human epidermis reconstructed by culture: Is it 'normal'? J Invest Dermatol 1986, 86, 181-6.

${ }^{8}$ Birch J and Branemark PI: The vascularisation of a free full thickness skin graft. Scand J Plast Reconstr Surg 1969, 3: 1-20.

${ }^{9}$ Cuono C, Langdon R, McGuire J: Use of cultured 
epidermal autografts and dermal allografts as skin replacement after burn injury. Lancet 1986 , i: $1123-4$.

${ }^{10}$ Yannas, IV et al.: Prompt, longterm functional replacement of skin. In Wise DL ed Burn Wound Coverings. CRC Press 1984: 28-33.

${ }^{11}$ Hefton JM et al.: Grafting of burn patients with allografts of cultured epidermal cells. Lancet 1983: ii, 428-30.

12 Faure M et al.: Growth and differentiation of human epidermal cultures used as auto- and allografts in humans. Br J Dermatol 1987, 116: 161-70.

${ }^{13}$ Lafferty KJ et al.: Thyroid allograft immunogenicity is reduced after a period in organ culture. Science 1975, 188: 259-61.

${ }^{14}$ Achauer BM et al.: Long-term skin allograft survival after short-term cyclosporin treatment in patients with massive burns. Lancet 1986, i, 14-15.

${ }^{15}$ Eisinger M: Regeneration of epidermis by cells grown in tissue culture. $\mathrm{J} \mathrm{Am} \mathrm{Acad} \mathrm{Dermatol}$ 1985, 12: 402-8.

${ }^{16}$ Auböck J: Personal Communication 1987. 